\title{
Melting Curve, Eutectic Point and Lindemann's Melting Temperatures of hcp Binary Alloys Studied Based on Debye-Waller Factors
}

\author{
Nguyen Cong Toan ${ }^{a}$, Dinh Quoc Vuong ${ }^{b}$ And Nguyen Van Hung ${ }^{a, *}$ \\ ${ }^{a}$ Department of Physics, VNU University of Science, \\ 334 Nguyen Trai, Thanh Xuan, Hanoi, Vietnam \\ ${ }^{b}$ bCam Pha Education $\&$ Training Department, \\ Cam Pha, Quang Ninh, Vietnam
}

Received: 18.01.2021 \& Accepted: 24.05.2021

\begin{abstract}
A method for studying the melting curves, eutectic point, and Lindemann's melting temperatures of hcp binary alloys is derived, based on the Debye-Waller factors given in terms of mean square displacement. Analytical expressions obtained for the considered quantities are based on Lindemann's criterion on melting. The mean square displacement is applied to calculate the ratio of the root mean square fluctuation in atomic positions around their equilibrium position in the lattice and atomic nearest neighbor distance. Using these results, the expression of the melting curve of hcp binary alloys is derived, which provides information on Lindemann's melting temperatures and eutectic point with respect to any proportion of hcp constituent elements. The proposed method allows to specify the binary alloy forms for the calculated atomic number of host and doping elements and their distribution in each elementary cell. Numerical results for the melting curve, eutectic point of $\mathrm{Cd}_{1-x} \mathrm{Zn}_{x}$, and for Lindemann's melting temperatures of several hcp binary alloys agree well with experiment.
\end{abstract}

topics: melting curve and eutectic point, Lindemann's melting temperature, the Debye-Waller factor, hcp binary alloys

\section{Introduction}

The atomic vibration theory has been successfully applied by Lindemann and others $[1-5]$ to the researches on melting. Lindemann's criterion on melting $[1,2]$ is based on the concept that the melting occurs when the ratio of the root mean square fluctuation (RMSF) in atomic positions around equilibrium lattice position and the atomic nearest neighbor distance reaches the threshold value. The validity of this criterion was tested experimentally [6]. The binary alloy phase diagrams have been measured [7].

Many efforts have been made to derive procedures for studying the melting problems. The phenomenological theory of the phase diagrams of the binary eutectic systems [8] has been developed to qualitatively show temperature-concentration diagrams of eutectic mixtures with the use of a Landautype approach. This approach involves a coupling between the liquid-solid transition orderparameters and a specific nonlinear dependence on concentration of the free-energy coefficients. Based on this theory, the eutectic point can be defined more generally as the minimum of the melting curve. Extended X-ray absorption fine structure
(EXAFS) [9] in studying melting is focused mainly on the Fourier transform magnitudes and cumulants of EXAFS. The melting curve of materials has been studied in experiments [10] and theory, where results in theory were obtained from quantum mechanics within the framework of density functional theory using the generalized gradient corrections. The purpose of these studies focused, however, mainly on the dependence of the melting temperature of single elements on pressure. In order to characterize the melting transition of solids, empirical rules [1, 11-13] have been applied as useful procedures in computer simulations without performing free energy calculations [14]. The mechanism for the solid-liquid phase transition that is based on Lindemann's criterion has been studied using a Monte-Carlo simulation [15]. The dependent pressure melting curves have also been computed with $a b$ initio molecular dynamics simulations for some single crystals [16].

A method of melting simulation (the shock melting method) has been proposed and it proved to be able to determine the melting curves based on the multi-scale shock technique and preheating and/or pressuring materials before shock [17]. By extensive molecular dynamics simulation of both 
two- and three-dimensional polydisperse LennardJones solids, it is shown that particles on the small or large limits of size distribution exhibit substantially different Lindemann ratio at melting [18]. The Debye-Waller factors (DWF) which appeared due to atomic vibrations play an important role in studying thermodynamic properties and some physical effects of materials [9, 19-22]. Therefore, they can be applied in creating procedures for solving the melting problems. An effort has been made to carry out such investigation [23], where the DWFs presented in terms of the mean square displacement (MSD, which is ratio $A / B$, where $A$ - root mean square, $B$ - distance) and the Lindemann parameters of several hexagonal close-packed (hcp) crystals, $\mathrm{Co}, \mathrm{Ru}$, and $\mathrm{Sc}$, have been studied using the lattice-dynamical model. Unfortunately, the results of this research are still not universal for providing the Lindemann melting temperatures of the considered crystals, especially for obtaining those of the hcp binary alloys.

The purpose of this work is to derive a method for the calculation and analysis of the melting curves, eutectic points, and Lindemann's melting temperatures of hcp binary alloys based on the DWF presented in terms of MSD. In Sect. 2, the analytical expressions meeting Lindemann's criterion on melting of the considered quantities are given. These include, for example, the expressions of the atomic MSD, the atomic mean square fluctuation (MSF), and then the ratio of the RMSF in atomic positions and the atomic nearest neighbor distance leading to the melting curves of hcp binary alloys. Worthy of noting is that the derived melting curve provides information about the Lindemann melting points of the binary hcp alloys. The model has such an advantage that it gives the melting points with respect to any proportion of the hcp constituent elements, as well as information on the possible eutectic melting and the eutectic point values (minimum melting point) of the considered binary alloys hcp (see numerical results).

Moreover, our model also provides the melting temperature of one hcp crystal as the limiting case when the atoms of another constituent element are taken out of the binary alloy. To specify the forms of hcp binary alloys, we create a method due to which the total atomic number, the numbers of the host and doping constituent element atoms, as well as their distributions in each hcp binary alloy elementary cell with respect to their different atomic proportions are determined. Numerical calculations have been carried out for several hcp binary alloys (see Sect. 3). The melting curves of hcp binary alloys calculated using the present theory are compared to their experimental phase diagrams [7] and the results for $\mathrm{Cd}_{1-x} \mathrm{Zn}_{x}$ are found to be in good and reasonable agreement with those taken from their experimental phase diagrams [7]. The conclusions and possible applicabilities of the derived theory are presented in Sect. 4 .

\section{Formalism}

To calculate the melting curve from which Lindemann's melting temperatures and eutectic point of a binary alloy are obtained, it is necessary to first calculate the RMSF in atomic positions around equilibrium lattice positions and the nearest neighbor distance [1-4]. This RMSF is often concerned with the atomic vibration whose amplitude is characterized by the MSD or DWF [19-24]. Therefore, we start our consideration from this DWF using its expression [24]:

$$
W=\frac{1}{2} \sum_{q}\left|\boldsymbol{K} \cdot \overline{\boldsymbol{u}}_{q}\right|^{2},
$$

where $\boldsymbol{K}$ is the scattering vector equaling a reciprocal lattice vector, and $\overline{\boldsymbol{u}}_{q}$ is the mean atomic vibration vector.

If each hcp binary alloy lattice cell contains $n$ atoms consisting of $s$ being the number of atoms of type 1 and $(n-s)$ as the atomic number of type 2 , then the averaged vibration atomic vector $\overline{\boldsymbol{u}}_{q}$ is given by

$$
\overline{\boldsymbol{u}}_{q}=\frac{s \boldsymbol{u}_{1 q}+(n-s) \boldsymbol{u}_{2 q}}{n} .
$$

The potential energy of an oscillator is equal to its kinetic energy so that the mean energy of atom $k$ vibrating with the wave number $q$ has the form

$$
\bar{\varepsilon}_{q}=M_{k}\left|\dot{\boldsymbol{u}}_{k q}\right|^{2} \text {. }
$$

Then, the mean energy of the crystal consisting of $N$ lattice cells is given by

$$
\begin{aligned}
& \bar{E}=\sum_{q} \bar{\varepsilon}_{q}= \\
& \sum_{q} N\left(s M_{1} \omega_{q}^{2}\left|\boldsymbol{u}_{1 q}\right|^{2}+(n-s) M_{2} \omega_{q}^{2}\left|\boldsymbol{u}_{2 q}\right|^{2}\right),
\end{aligned}
$$

where $M_{1}, M_{2}$ are the masses of atoms of types 1 and 2 , respectively, and $u_{2 q}$ is related with $u_{1 q}$ in the following way [20]:

$$
u_{2 q}=m u_{1 q}, \quad m=\frac{M_{1}}{M_{2}} .
$$

Now, using (2) and (5), the mean energy for the atomic vibration of the $q$-th lattice mode is obtained, i.e.,

$$
\bar{\varepsilon}_{q}=N \omega_{q}^{2}\left|\boldsymbol{u}_{1 q}\right|^{2}\left(s M_{1}+(n-s) M_{2} m^{2}\right) .
$$

Comparing (6) with the phonon energy of $n$ atoms in a lattice cell, calculated with

$$
\bar{\varepsilon}_{q}=n \hbar \omega_{q}\left(\bar{n}_{q}+\frac{1}{2}\right)
$$

where $\bar{n}_{q}$ - the mean number of phonons, we get that

$$
\left|\boldsymbol{u}_{1 q}\right|^{2}=\frac{n \hbar}{N M_{1} \omega_{q}} \frac{\bar{n}_{q}+\frac{1}{2}}{s+m(n-s)} .
$$

Using (2) and (4), the square amplitude of the mean atomic vibration mode can be provided for the $q$-th lattice. Namely,

$$
\begin{aligned}
& \left|\overline{\boldsymbol{u}}_{q}\right|^{2}=\frac{1}{n^{2}}(s+m(n-s))^{2}\left|\boldsymbol{u}_{1 q}\right|^{2}= \\
& \quad \frac{(s+m(n-s))\left(\bar{n}_{q}+\frac{1}{2}\right)}{N M_{1} \omega_{q} n} .
\end{aligned}
$$


In order to determine the DWF presented in terms of MSD, we should calculate the contribution of each polarization, take (9) into account and then use (8). The MSD given in (1) when all three polarizations are included, resulted in

$$
W=\frac{\hbar}{2 N M_{1} n} \sum_{q} K^{2} \frac{(s+m(n-s))\left(\bar{n}_{q}+\frac{1}{2}\right)}{\omega_{q}}
$$

where $K=|\boldsymbol{K}|$. The sum over $q$ in (10) can be transformed into the corresponding integral and applying it in the area of high temperatures $\left(T \gg \theta_{\mathrm{D}}\right)$ with respect to melting with the Debye temperature $\theta_{\mathrm{D}}$, one obtains the MSD or DWF expression given by (10). This is

$$
W=\frac{3 \hbar^{2} K^{2} T}{2 n k_{\mathrm{B}} \theta_{\mathrm{D}}^{2}} \frac{s M_{2}+(n-s) M_{1}}{M_{1} M_{2}} .
$$

Note that (11) is linearly proportional to the temperature $T$, as already mentioned [9, 19-24].

Based on the mean crystal lattice energy obtained in the form of

$$
\bar{E}=\sum_{k, i} M_{k}\left|\dot{U}_{k i}\right|^{2}=\sum_{k, i} \sum_{q} M_{k} \omega_{q}^{2}\left|U_{k i q}\right|^{2},
$$

where $U_{k i}$ is the atomic fluctuation function, and on the energy given by (4) as well as on the above results, the atomic MSF is determined as

$$
\begin{aligned}
& \frac{1}{N} \sum_{i}\left|U_{2 i}\right|^{2}=m^{2} \sum_{q}\left|\boldsymbol{u}_{1 q}\right|^{2}= \\
& \frac{6 W}{K^{2}} \frac{n^{2} m^{2}}{(s+m(n-s))^{2}} .
\end{aligned}
$$

With the use of the expression of DWF (11), (13) can be transformed into

$$
\frac{1}{N} \sum_{i}\left|U_{2 i}\right|^{2}=\frac{9 \hbar^{2} T}{k_{\mathrm{B}} M_{1} \theta_{\mathrm{D}}^{2}} \frac{n m^{2}}{s+m(n-s)} .
$$

Hence, when $T \gg \theta_{\mathrm{D}}$, the MSF in atomic positions that is determined by (14) bases on the DWF given by (11). This obtained MSF is linearly proportional to the temperature $T$.

Therefore, at a given temperature $T$, the quantity $R$ is defined as:

$$
R=\frac{1}{d} \sqrt{\frac{9 \hbar^{2} T}{k_{\mathrm{B}} M_{1} \theta_{\mathrm{D}}^{2}} \frac{n m^{2}}{s+m(n-s)}},
$$

i.e., as the ratio of the RMSF in atomic positions around the equilibrium position in the lattice and the atomic nearest neighbor distance $d$.

This expression for $R$ contains the parameter $s$ describing the number of doping atoms in the host element and the parameter $m$ concerning the atomic mass $M_{1}$ of element 1 and the atomic mass $M_{2}$ of element 2 composing the hcp binary alloys.

Based on Lindemann's criterion on melting, the binary alloy will be melted when $R$ given by (15) reaches the threshold value $R_{\mathrm{m}}$, namely

$$
R_{\mathrm{m}}^{2}=\frac{1}{N d^{2}} \sum_{i}\left|U_{2 i}\right|^{2} \text {. }
$$

Then, Lindemann's melting temperature $T_{\mathrm{m}}$ of the hcp binary alloy is defined as:

$$
\begin{aligned}
& T_{\mathrm{m}}=\frac{s M_{2}+(n-s) M_{1}}{9 n m} \chi, \\
& \chi=\frac{k_{\mathrm{B}} \theta_{\mathrm{D}}^{2} d^{2}}{\hbar^{2}} R_{\mathrm{m}}^{2} .
\end{aligned}
$$

In order to determine Lindemann's melting temperature of the hcp binary alloy (17), it is very important to specify $\chi(18)$ at first, which contains the threshold value $R_{\mathrm{m}}$ of the ratio of the RMSF in atomic positions around the equilibrium lattice positions and the nearest neighbor distance at the melting.

To simplify the computation, we have to introduce an averaging procedure in the present approach. The average of $\chi$ cannot base directly on $\chi_{1}$ and/or $\chi_{2}$ because its form (18) contains $R_{m}^{2}$, i.e., the second order of $R_{m}$, while the other averages in (18) are based on the first order of the displacement as shown in (17). For this reason, we should find the averages of the individual $\sqrt{\chi_{i}}$ for $i=1,2$, and further use them to obtain

$$
\chi=\frac{1}{n}\left(s \sqrt{\chi_{1}}+(n-s) \sqrt{\chi_{2}}\right)^{2} .
$$

The above expression contains $\chi_{1}$ - for the first element, and $\chi_{2}$ - for the second element of the hcp binary alloy, with the following limiting values:

$$
\begin{aligned}
& \chi_{2}=9 T_{\mathrm{m}(2)} / M_{2}, \quad s=0 ; \\
& \chi_{1}=9 T_{\mathrm{m}(1)} / M_{1}, \quad s=n .
\end{aligned}
$$

Here, $T_{\mathrm{m}(1)}$ and $T_{\mathrm{m}(2)}$ denote the melting temperatures of the first or doping element and of the second or host element, respectively, composing the binary alloy.

As a result, we have obtained the melting temperature of hcp binary alloys, actually, by calculating the ratio of the RMSF in atomic positions on the equilibrium lattice positions and nearest neighbor distance, given by (15).

The eutectic point is calculated using the condition for the minimum of the melting curve, i.e.,

$$
\frac{\mathrm{d} T_{\mathrm{m}}}{\mathrm{d} x}=0 .
$$

The determination of the melting curves allows to obtain Lindemann's melting temperatures (using (17)-(20)) and eutectic points (using (21)) of hcp binary alloys with respect to any proportions of their constituent elements. The eutectic isotherm certainly is the one for which $T$ equals the eutectic temperature $T_{\mathrm{e}}$.

\section{Numerical results and discussions}

\subsection{Forms of hep binary alloys}

To specify the forms of hcp binary alloys, we propose a method to determine the total atomic number $n$ and the numbers of the host $\left(n_{\mathrm{H}}\right)$ and doping $\left(n_{\mathrm{D}}\right)$ constituent element atoms. Also, their distributions in each elementary cell (EC) of hcp 


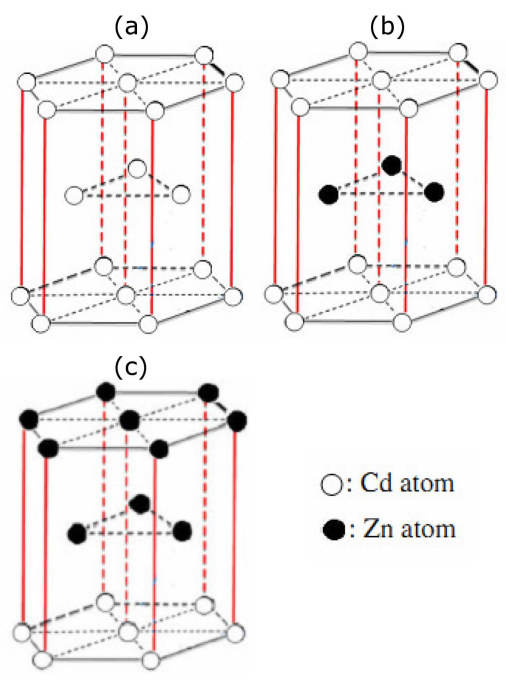

Fig. 1. Possible distributions of atoms of the host element $\mathrm{Cd}$ and the doping element $\mathrm{Zn}$ in each hcp EC providing their binary alloys forms for (a) $\mathrm{Cd}_{1.0} \mathrm{Zn}_{0.0}$ with $s=0$ (i.e., no $\mathrm{Cd}$ atom in $\mathrm{EC}$ is doped by $\mathrm{Zn}$ atom), (b) $\mathrm{Cd}_{0.5} \mathrm{Zn}_{0.5}$ with $s=3$ (i.e., $3 \mathrm{Cd}$ atoms inside the EC are doped by $\mathrm{Zn}$ atoms), and (c) $\mathrm{Cd}_{0.25} \mathrm{Zn}_{0.75}$ with $s=4.5$ (i.e., 3 atoms inside and all atoms in the top surface of $\mathrm{EC}$ of $\mathrm{Cd}$ are doped by $\mathrm{Zn}$ atoms), where $s=n_{\mathrm{Zn}}$.

\section{TABLE I}

Distribution of atomic number $n_{\mathrm{Cd}}=n-s$ of the host constituent element $\mathrm{Cd}$ and the one $\left(n_{\mathrm{Zn}}=s\right)$ of the doping constituent element $\mathrm{Zn}$ composing the binary alloy $\mathrm{Cd}_{1-x} \mathrm{Zn}_{x}$ with respect to the proportion $x$ of the doping element $\mathrm{Zn}$ in these binary alloys, where $s=n_{\mathrm{Zn}}$.

\begin{tabular}{l|c|c|c}
\hline \hline hcp binary alloys & $x$ & $n_{\mathrm{Cd}}$ & $n_{\mathrm{Zn}}$ \\
\hline $\mathrm{Cd}_{0.92} \mathrm{Zn}_{0.08}$ & 0.08 & 5.5 & 0.5 \\
$\mathrm{Cd}_{0.75} \mathrm{Zn}_{0.25}$ & 0.25 & 4.5 & 1.5 \\
$\mathrm{Cd}_{0.5} \mathrm{Zn}_{0.5}$ & 0.50 & 3.0 & 3.0 \\
$\mathrm{Cd}_{0.25} \mathrm{Zn}_{0.75}$ & 0.75 & 1.5 & 4.5 \\
$\mathrm{Cd}_{0.08} \mathrm{Zn}_{0.92}$ & 0.92 & 0.5 & 5.5
\end{tabular}

binary alloy with respect to their different atomic proportion $x$ can be determined with our method. Figure 1a shows that there are $1 / 6$ atom on each of 12 vertexes, 3 atoms inside the $\mathrm{EC}$, and $1 / 2$ atom in the top and in the bottom surfaces of each EC. Therefore, the total number of atoms in each hcp EC is $n=6$ and it consists of the atomic number $n_{\mathrm{H}}$ for the host constituent element $\mathrm{H}=\mathrm{Cd}$ and the atomic number $n_{\mathrm{D}}$ for the doping element $\mathrm{D}=\mathrm{Zn}$, depending on the proportion $x$ of the doping element. These numbers have been calculated and written in Table I for binary alloys belonging to $\mathrm{Cd}_{1-x} \mathrm{Zn}_{x}(\mathrm{H}=\mathrm{Cd}, \mathrm{D}=\mathrm{Zn})$. The proportion $x$ is based on $\mathrm{Zn}$ doped.

For each of the hcp binary alloys, there are several possibilities of $\mathrm{Zn}$ atoms doped in the EC of $\mathrm{Cd}$ providing the forms of their binary alloys.
Figure 1 illustrates (a) the limiting case described by $\mathrm{Cd}_{1.0} \mathrm{Zn}_{0.0}$ where no $\mathrm{Cd}$ atom in the $\mathrm{EC}$ is doped by the $\mathrm{Zn}$ atom, (b) the binary alloy $\mathrm{Cd}_{0.5} \mathrm{Zn}_{0.5}$ obtained when $3 \mathrm{Cd}$ atoms inside the $\mathrm{EC}$ are doped by the $\mathrm{Zn}$ atoms, and (c) the binary alloy $\mathrm{Cd}_{0.25} \mathrm{Zn}_{0.75}$ obtained when $3 \mathrm{Cd}$ atoms inside and all atoms in the top or in the bottom surface of the EC are doped by the $\mathrm{Zn}$ atoms.

Note that the forms of hcp binary alloys in Table I have been given based on the total atomic number $n$ and the atomic numbers $n_{\mathrm{Cd}}$ and $n_{\mathrm{Zn}}$ of the host (Cd) and doping (Zn) constituent elements, respectively, in each hcp EC with respect to the proportion $x$ of the doping element $\mathrm{Zn}$ in these binary alloys.

\subsection{Melting curves, eutectic points and}

Lindemann's melting temperatures

The numerical results calculated using the present theory are focused mainly on the melting curves that provide information on Lindemann's melting temperatures of hcp eutectic binary alloys with respect to any proportion of the constituent elements and on the eutectic points of binary alloys composed by two different hcp elements. The eutectic isotherm is apparently $T=T_{\mathrm{e}}$. All input data have been taken from [25].

Figure 2 illustrates the melting curves providing information on Lindemann's melting temperatures and eutectic points of eutectic binary alloys (a) $\mathrm{Cd}_{1-x} \mathrm{Zn}_{x}$ and (b) $\mathrm{Zn}_{1-x} \mathrm{Tl}_{x}$ that are calculated using the present theory. The results for $\mathrm{Cd}_{1-x} \mathrm{Zn}_{x}$ are found to be in good and reasonable agreement with the experimental values taken from its experimental phase diagram [7]. The melting curves respond to those of the experimental phase diagrams [7] where the eutectic temperature of $\mathrm{Cd}_{1-x} \mathrm{Zn}_{x}$ is smaller than the melting temperatures of the host element $\mathrm{Cd}$ and the doping element $\mathrm{Zn}$ (Fig. 2a) and also where the eutectic temperature of $\mathrm{Zn}_{1-x} \mathrm{Tl}_{x}$ is smaller than the melting temperatures of the host elements $\mathrm{Zn}$ and the doping element Tl (Fig. 2b).

Figure 3 illustrates the melting curves providing information on Lindemann's melting temperatures and eutectic points of eutectic binary alloys (a) $\mathrm{Cd}_{1-x} \mathrm{Ti}_{x}$ and (b) $\mathrm{Co}_{1-x} \mathrm{Zn}_{x}$. The results are obtained using the present theory for the cases where the eutectic temperatures are minimal melting temperatures equaling the melting temperature of the host element $\mathrm{Cd}$ for $\mathrm{Cd}_{1-x} \mathrm{Ti}_{x}$ (Fig. 3a) and of the doping element $\mathrm{Zn}$ for $\mathrm{Co}_{1-x} \mathrm{Zn}_{x}$ (Fig. 3b).

Notably, the results presented in Figs. 2 and 3 show that Lindemann's melting temperatures of the considered hcp binary alloys (i) change from the melting temperatures of the pure host elements (when the whole elementary cell is occupied by host atoms) to those of the binary alloys of different increasing proportions $x$ (when the doping elements are $\mathrm{Zn}, \mathrm{Tl}$, and $\mathrm{Ti}, \mathrm{Zn}$, respectively), and (ii) end 

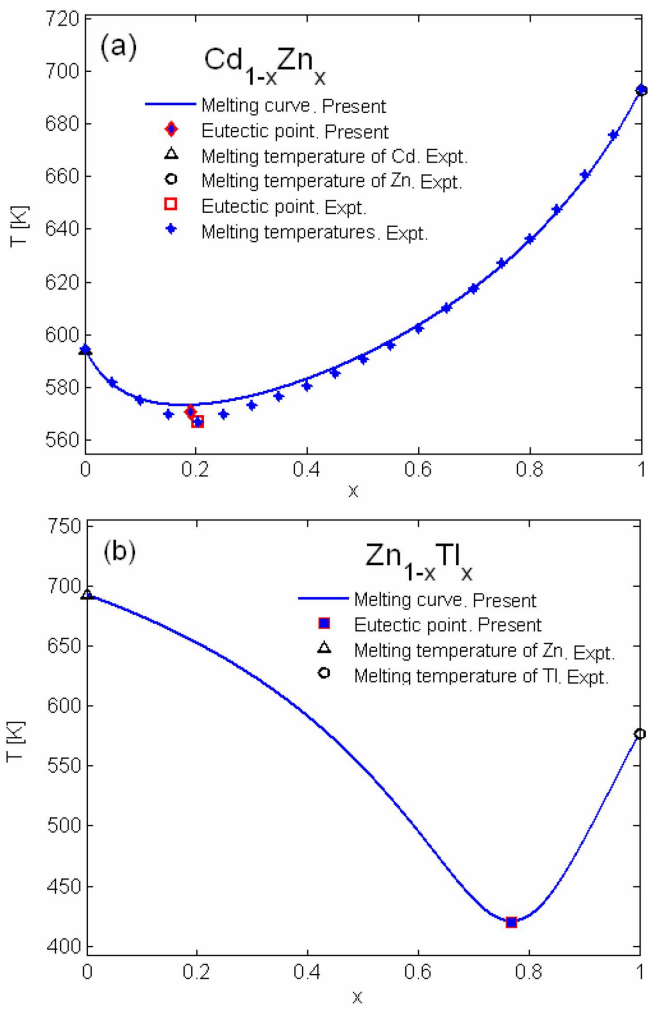

Fig. 2. Melting curves providing information on Lindemann's melting temperatures and eutectic points calculated using the present theory for eutectic binary alloys (a) $\mathrm{Cd}_{1-x} \mathrm{Zn}_{x}$ and (b) $\mathrm{Zn}_{1-x} \mathrm{Tl}_{x}$ compared to the experimental melting temperatures [25] for $\mathrm{Cd}, \mathrm{Zn}, \mathrm{Tl}$ and to the values taken from the experimental phase diagram [7] for $\mathrm{Cd}_{1-x} \mathrm{Zn}_{x}$.

at the melting temperatures of these purely doping elements (when the atoms of these doping elements occupy the whole elementary cell). In addition, Fig. 2 shows the rate at which Cd (Fig. 2a) and Zn (Fig. 2b) become softer after they were mixed by the doping elements $\mathrm{Zn}$ and $\mathrm{Tl}$, respectively. This softening takes place because the melting temperatures of $\mathrm{Cd}$ and $\mathrm{Zn}$ decrease up to their eutectic point. In turn, the hardening of $\mathrm{Cd}$ and $\mathrm{Zn}$ takes place after passing the eutectic point because their melting temperatures increase.

Figure 3a for $\mathrm{Cd}_{1-x} \mathrm{Ti}_{x}$ shows the rate at which the host element $\mathrm{Cd}$ becomes harder after it was doped by Ti. It happens due to the increase of its melting temperature. In turn, Fig. $3 \mathrm{~b}$ for $\mathrm{Co}_{1-x} \mathrm{Zn}_{x}$ shows the rate at which the host element Co becomes softer after it was doped by Zn. It happens due to the decrease of its melting temperature. The above mentioned property that the hcp element becomes either harder or softer after it was mixed by another hcp element to be a binary alloy can be attributed to the fact that the atomic bonding becomes either tighter or more weakly, respectively, in the binary alloy depending on the proportion of the doping element in the binary alloy.
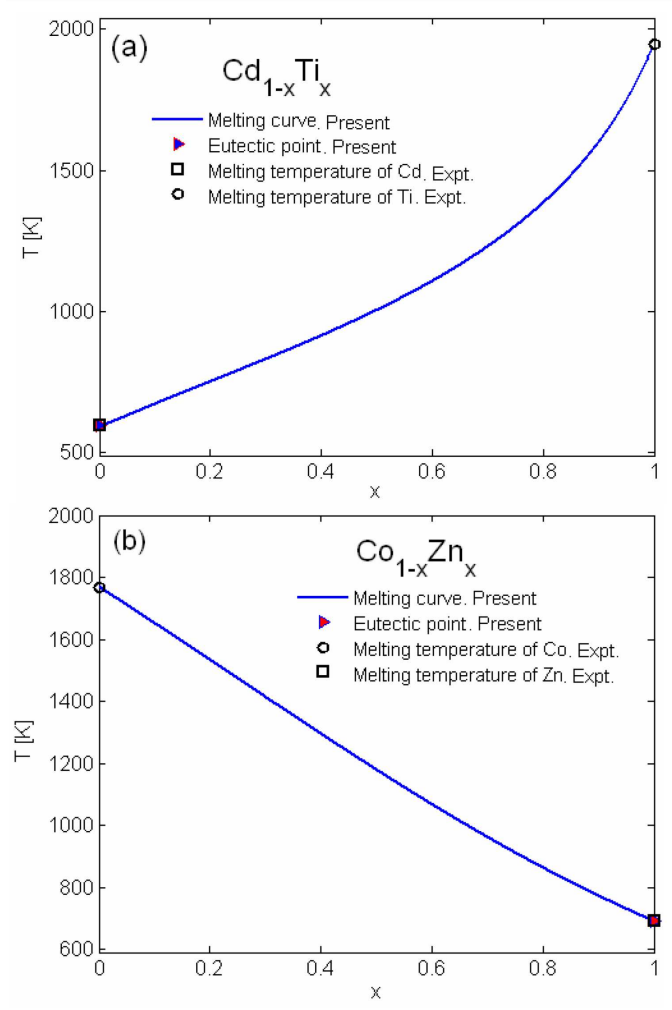

Fig. 3. Melting curves providing information on Lindemann's melting temperatures and eutectic points calculated using the present theory for eutectic binary alloys (a) $\mathrm{Cd}_{1-x} \mathrm{Ti}_{x}$ and (b) $\mathrm{Co}_{1-x} \mathrm{Zn}_{x}$ compared to the experimental melting temperatures [25] of $\mathrm{Cd}, \mathrm{Ti}, \mathrm{Co}$, and $\mathrm{Zn}$.

TABLE II

Lindemann's melting temperatures $T_{\mathrm{m}}[\mathrm{K}]$ taken from the melting curve calculated using the present theory for some hcp binary alloys belonging to $\mathrm{Cd}_{1-x} \mathrm{Zn}_{x}$ (see Fig. 2a) with respect to their proportion $x$ of element $\mathrm{Zn}$ doped in these binary alloys compared to the experimental values taken from the experimental phase diagram [7].

\begin{tabular}{l|c|c|c}
\hline $\begin{array}{c}\text { hcp binary } \\
\text { alloys }\end{array}$ & $\begin{array}{c}\text { Proportion } x \\
\text { of } \mathrm{Zn}\end{array}$ & $\begin{array}{c}T_{\mathrm{m}}[\mathrm{K}] \\
\text { this work }\end{array}$ & $\begin{array}{c}T_{\mathrm{m}}[\mathrm{K}] \\
\text { exp. [7] }\end{array}$ \\
\hline $\mathrm{Cd}_{0.92} \mathrm{Zn}_{0.08}$ & 0.08 & 576.7 & 576.0 \\
$\mathrm{Cd}_{0.75} \mathrm{Zn}_{0.25}$ & 0.25 & 573.0 & 570.0 \\
$\mathrm{Cd}_{0.5} \mathrm{Zn}_{0.5}$ & 0.50 & 592.0 & 590.5 \\
$\mathrm{Cd}_{0.25} \mathrm{Zn}_{0.75}$ & 0.75 & 626.0 & 627.0 \\
$\mathrm{Cd}_{0.08} \mathrm{Zn}_{0.92}$ & 0.92 & 664.2 & 664.3
\end{tabular}

Moreover, it is shown in Figs. 2 and 3 that the calculated melting temperatures of the host constituent element at $x=0$ and of the doping constituent element at $x=1$ (the limiting cases of binary alloys), i.e., the melting temperatures of hcp constituent elements, agree well with their experimental values [25]. The obtained eutectic point of $\mathrm{Cd}_{1-x} \mathrm{Zn}_{x}$ (Fig. 2a) is close to the experimental result [7]. Moreover, the localizations of the 
calculated eutectic points of hcp binary alloys also respond to those predicted by the phenomenological theory for the phase diagrams of the binary eutectic systems [8] and in experiment [7].

Lindemann's melting temperatures taken from the melting curve calculated using the present theory for binary alloys belonging to $\mathrm{Cd}_{1-x} \mathrm{Zn}_{x}$ (Fig. 2a) - with respect to their proportions $x$ of $\mathrm{Zn}$ doped in $\mathrm{Cd}$ - are found to be in good and reasonable agreement with the experimental values taken from the experimental phase diagram [7]. Some of these results are given in Table II.

According to the present theory, the eutectic melting of hcp binary alloys based on providing their eutectic points (where some values of these eutectic points are presented in Figs. 2 and 3) are found and are in good and reasonable agreement with the experimental values $[7,25]$.

\section{Conclusions}

In this work, a method is derived for the calculation and analysis of the melting curves, eutectic points and Lindemann's melting temperatures of binary alloys composed by any hcp elements based on the Debye-Waller factors presented in terms of mean square displacement.

Analytical expressions of the considered quantities have been derived based on Lindemann's criterion on melting. They are simplified for the numerical calculations.

Our method has the advantage of providing Lindemann's melting temperatures of hcp binary alloys respective to any proportion of their constituent elements that compose these binary alloys. Further, also the melting temperature of a hcp constituent element can be obtained when the atoms of another constituent element are taken out of the binary alloy. This illustrates the possible eutectic melting of hcp binary alloys based on providing their eutectic points.

Our method allows to determine the total atomic number, the numbers of the host and doping constituent elements atoms, as well as their distributions in each hcp binary alloy elementary cell with respect to their different atomic proportions. Therefore, one can quite well specify the binary alloys forms.

We have successfully applied our method to study several hcp binary alloys for which the obtained results show the rate at which hcp's binary alloy atoms become either more tightly or more weakly bonded. It simply means that the host element becomes either harder or softer after it was mixed by the doping element to be the hcp binary alloy based on either the increase or decrease of the melting temperature with respect to increasing the proportion of the doping element in the hcp binary alloy. This behavior may be useful for technological applications.
The good agreement of the calculated melting curves and eutectic points of $\mathrm{Cd}_{1-x} \mathrm{Zn}_{x}$ with their experimental phase diagrams, as well as of the calculated Lindemann's melting temperatures of several hcp binary alloys with their measured values illustrate the advantage and efficiency of the present theory for forecasting the melting curves, the eutectic points, Lindemann's melting temperatures, and the eutectic isotherms of hcp binary alloys including those which have not yet been investigated.

\section{Acknowledgments}

The authors thank J.J. Rehr and P. Fornasini, as well as kindly acknowledge the Editors and Reviewer for their useful comments and suggestions.

\section{References}

[1] F.A. Lindemann, Z. Phys. 11, 609 (1910).

[2] J.J. Gillvarry, Phys. Rev. 102, 308 (1956).

[3] N. Snapipiro, Phys. Rev. B 1, 3982 (1970).

[4] H.H. Wolf, R. Jeanloz, J. Geophys. Res. 89, 7821 (1984).

[5] Z.H. Jin, P. Gumbsch, K. Lu, E. Ma, Phys. Rev. Lett. 87, 055703 (2001).

[6] C.J. Martin, D.A. O'Connor, J. Phys. C Solid State Phys. 10, 3521 (1977).

[7] Binary Alloy Phase Diagrams, 2nd ed., Eds. T.B. Massalski, H. Okamoto, P.R. Subramanian, L. Kacprzak, ASM International Materials Parks, 1990.

[8] D. Machon, P. Toledano, G. Krexner, Phys. Rev. B 71, 024110 (2005).

[9] E.A. Stern, P. Livins, Zhe Zhang, Phys. Rev B 43, 8850 (1991).

[10] D. Alfè, L. Vočadlo, G.D. Price, M.J. Gillan, J. Phys. Condens. Matter 16, S937 (2004).

[11] H. Löwen, T. Palberg, R. Simon, Phys. Rev. Lett. 70, 1557 (1993).

[12] H. Löwen, Phys. Rep. 237, 249 (1994).

[13] F.H. Stillinger, Science 267, 1935 (1995).

[14] Y. Zhou, M. Karplus, K.D. Ball, R.S. Berry, J. Chem. Phys. 116, 2323 (2002).

[15] C. Chakravaty, P.G. Debenedetti, F.H. Stillinger, J. Chem. Phys. 126 , 204508 (2007).

[16] G. Robert, P. Legrand, P. Arnault, N. Desbiens, J. Clerouin, Phys. Rev. E 91, 033310 (2015).

[17] Zhong Li-Liu, Xiu-Lu Zhang, Ling-Cang Cai, J. Chem. Phys. 143, 114101 (2015).

[18] S. Sarkar, C. Jana, B. Bagchi, J. Chem. Sci. 129, 833 (2017). 
[19] N.V. Hung, J.J. Rehr, Phys. Rev. B 56, 43 (1997).

[20] M. Daniel, D.M. Pease, N. Van Hung, J.I. Budnick, Phys. Rev. B 69, 134414 (2004).

[21] N.V. Hung, C.S. Thang, N.C. Toan, H.K. Hieu, Vacuum 101, 63 (2014).

[22] S. a Beccara, G. Dalba, P. Fornasini, R. Grisenti, F. Pederiva, A. Sanson, D. Diop, F. Rocca, Phys. Rev. B 68, 140301(R) (2003).
[23] A. Ramanand, R. Ramji Rao, Can. J. Phys. 58, 384 (1980).

[24] J.M. Ziman, Principles of the Theory of Solids, Cambridge University Press, London 1972.

[25] Ch. Kittel, Introduction to Solid State Physics, 7th ed., Wiley, New York 1996. 